\title{
Biomarkers for Diagnosis and Prognosis of Acute Heart Failure
}

\author{
Rajiv Choudhary $\cdot$ Salvatore Di Somma $\cdot$ \\ Alan S. Maisel
}

Published online: 7 March 2013

(c) Springer Science+Business Media New York 2013

\begin{abstract}
High mortality, morbility and hospitalization as a result of acute heart failure (AHF) represents an increasing public health dilemma. A prompt and appropriate therapeutic approach in the management of AHF has been demonstrated to be of great importance in reducing patient mortality and in-hospital length of stay. As consequence, at the moment of patient presentation, it is of great importance to make a fast and accurate diagnosis and risk stratification. Although an global clinical evaluation is mandatory, in some cases the signs and instrumental findings are not sufficient for a complete patient's diagnosis and risk stratifications. Multiple studies demonstrated that biomarkers assessment plus clinical judgement provide additional diagnostic and prognostic value in AHF patients. Moreover, data from the literature demonstrated the utility of a multimarkers approach in patients with heart failure in order to ameliorate diagnostic and prognostic accuracy. This paper is dedicated to addressing the actual state of the art on the utility in the management of acute heart failure of the following biomarkers: natriuretic peptides, procalcitonin, MRproADM, copeptine, neutrophil gelatinase associated lipocalin and galectin 3 .
\end{abstract}

Keywords Acute heart failure - Diagnosis - Prognosis · Clinical judgement $\cdot$ Risk stratification $\cdot$ Shortness of

R. Choudhary · A. S. Maisel

Veterans Affairs San Diego Healthcare System, 3350 La Jolla

Village Drive, San Diego, CA 92122, USA

S. Di Somma $(\bowtie)$

Department of Medical-Surgery Sciences and Traslational Medicine, Emergency Department Sant'Andrea Hospital, Postgraduate School of Emergency Medicine, University La Sapienza Rome, Rome, Italy

e-mail: salvatore.disomma@uniroma1.it breath $\cdot$ Pneumonia $\cdot$ Mortality $\cdot$ Morbility $\cdot$ In hospital length of stay $\cdot$ Follow-up $\cdot$ Kidney injury . Rehospitalization · Biomarkers · BNP · NTproBNP · MRproAdrenomedullin · Copeptin · Neutrophil gelatinase associated lipocalin · Galectin 3

\section{Introduction}

Acute heart failure (AHF) is a growing epidemic affecting millions worldwide. In the USA, AHF carries a high re-admission $(29.6 \%)$ rate and a high short-term mortality $(8.6 \%)$ rate [1]. The AHF registries have shown that the patients are usually age $>65$ years, and have multiple comorbidities which are poorly controlled possibly due to dietary and/or pharmacological non-compliance. This results in re-admissions and longer in-hospital stays [2]. With a population estimated to have twice the number of older individuals in the next two decades, effective management and treatment of AHF is imperative in order to cut growing health-care costs and reduce the burden of disease $[2,3]$. Moreover, the rapid worsening of symptoms as seen in patients with AHF, in conjunction with involvement of other organs triggered by a failing heart, make it difficult to timely and appropriately treat these patients [4]. A large number of AHF patients initially present through the ED, and the majority of these patients have dyspnea which, on the basis of clinical examination, is often difficult to distinguish from non-cardiac dyspnea delaying treatment and worsening heart failure (HF) [5]. With the growing body of evidence concentrating on various methods for tackling the task at hand, biomarkers have been the focus of much attention and gaining popularity in the diagnosis and prognosis of patients with AHF. The utility of natriuretic peptides in identifying patients with cardiac from 
non-cardiac dyspnea has made them effective tools in the arsenal of AHF diagnosis and treatment. Furthermore, several other biomarkers such as troponins, procalcitonin and NGAL have provided robust evidence in diagnosing and guiding therapy in AHF patients. This chapter will provide a brief description of the aforementioned biomarkers as well as newer emerging biomarkers such as Copeptin and Galectin 3 with promising potential in aiding diagnosis and risk stratification of AHF patients and possibly laying the framework for biomarker guided multimarker strategy.

\section{Definition and Classification}

Clinically AHF can be defined as a syndrome involving rapid or gradual worsening of symptoms and signs secondary to abnormal cardiac structure or function leading to pump failure and inability of the heart to meet tissue oxygen demands $[2,4,6]$. Clinical signs used to diagnose $\mathrm{HF}$ include, jugular venous distension and elevated jugular venous pulse, third heart sound (S3), and chest x-ray imaging to look for pulmonary venous congestion. Although these tests carry high specificity, they lack sensitivity and diagnostic accuracy.

Patients with AHF can be classified into two sub-groups namely de novo or acute decompensated heart failure (ADHF) based on clinical manifestations of their symptoms and signs. Those with new onset HF secondary to an inciting event such as MI constitute de novo HF; whereas those patients with worsening of pre-existing chronic HF resulting in a decompensated state are said to have ADHF $[3,6,7]$. Furthermore, patients with chronic HF can be further classified based on their ejection fraction (prognostic marker of ventricular function) into those with preserved EF (HFpEF) and those with reduced EF (HFrEF). Systolic HF is comprised of patients with EF $<35 \%$, such patients tend to be older and are most likely to have hypertension and atrial fibrillation than CAD. Diastolic HF is comprised of patients with $\mathrm{HFpEF}$ including high $\mathrm{LV}$ filling pressures and impaired ventricular relaxation $[6,7]$.

\section{Pathophysiology}

The AHF patients often have decreased tissue perfusion due to reduced cardiac output and increased tissue congestion due to an elevated pulmonary capillary wedge pressure [8]. According to the AHF registry database (AHEAD), in patients with de novo HF, $51.3 \%$ had acute coronary syndrome; whereas in patients with ADHF, $36.9 \%$ had chronic coronary artery disease. Other etiological factors predisposing to AHF included hypertension, arrhythmias and valvular disorders [9]. In patients with new-onset HF or de novo HF is a result of an initial inciting event such as myocardial infarction, resulting in decreased left ventricular contractility and poor cardiac output. Suppressed left ventricular contractility often results in elevated left ventricular end diastolic pressure and pulmonary congestion leading to dyspnea, the chief complaint of patients presenting with HF [10]. In older patients with multiple comorbidities, this phenomenon is aggravated by the presence of stiff arteries leading to an increase in afterload and a further worsening of pulmonary dyspnea. Such patients with chronic worsening of their HF present in an acute decompensated state with increased dyspnea and fluid overload. Studies have shown that the injudicious use of diuretics in such patients often has poor outcomes due to resulting renal insufficiency [11]. The inability of the body to maintain euvolemia (normal fluid status) results in the activation of certain compensatory mechanisms, namely activation of the renin-angiotensinaldosterone system, sympathetic response and activation of the inflammatory cascade [12]. These pathways function synchronously to maintain fluid status and can lead to further worsening of $\mathrm{HF}$ in the long run.

\section{Ideal Biomarker in Acute Heart Failure}

The role of an ideal biomarker in patients with HF has evolved significantly over the past decade. Since the first proposed outline put forth by Morrow and Lemos [13] researchers have delineated several criteria a novel biomarker must fulfill in order to provide an accurate estimation of ongoing disease severity. An ideal biomarker must be highly sensitive and specific and reflect abnormal physiology and biochemistry, moreover, biomarkers must be cost-effective, have a low coefficient of variation and must be usable across diverse groups. Biomarkers must also be able to provide prognostic information and their levels must correlate with disease severity and reflect changes to HF treatment and therapy [14]. As the number of biomarkers utilized in the diagnosis and prognosis of AHF patients grows substantially, a single biomarker meeting all of the mentioned criteria is unrealistic, thus studies have shown that in order to achieve maximum utility of biomarkers, a multi-marker paradigm in diagnosis and treatment of AHF must be considered.

\section{Natriuretic Peptides}

The natriuretic peptides (NP) consist of circulating proteins released in direct response to increased pressure and volume overload in the ventricles [15]. Studies have shown that NPs not only provide valuable information in allowing 
the identification of patients with cardiac dyspnea from non-cardiac dyspnea but also provide significant prognostic information on short-term mortality and treatment outcomes [16]. The NP levels have also been used to titrate treatment in AHF and provide a better understanding of the ongoing physiological processes seen in patients with AHF. The NPs include atrial natriuretic peptide (ANP), B-type or brain natriuretic peptide (BNP), C-type and recently isolated D-type natriuretic peptide [17].

The ANP, as the name suggests, is mainly secreted from the atrial myocytes also in direct response to stretch. With a 99-26 amino acid structure, ANP, once in the circulation, leads to kaliuresis, natriuresis, suppression of the reninangiotensin-aldosterone system (RAAS) and sympathetic response [15-17]. Studies assessing the regulation and release of NPs have shown that BNP is superior to the other NPs mainly due to its longer half-life and possibly explained by a lack of affinity to clearance receptors. Furthermore, the nature of BNP release, which is in short bursts in direct response to ventricular volume and pressure from secretory granules, results in a rapid release into the circulation [18].

The BNP was initially isolated from the porcine brain in 1981 by de Bold et al. [19]. In humans, BNP consists of a 32 amino acid structure and is secreted into the circulation along with its inactive N-terminal fragment with a 76 amino acid structure $[17,18]$. The BNP mRNA up-regulation occurs in response to increasing left ventricular pressure and volume resulting in myocyte stretch. This leads to formation of the precursor propeptide proBNP, which is then cleaved by serine proteases to give the biologically active BNP and inactive N-terminal fragment of BNP. Once in the circulation for almost 21 min, BNP binds clearance receptors, namely NPRA and neutral endopeptidases, and is renally cleared [20]. The inactive N-terminal fragment of BNP propeptide is present in the plasma for a longer time $(70 \mathrm{~min})$ due its poor affinity to clearance receptors, moreover, researchers have shown that NTproBNP levels significantly correlate with HF disease severity and can also be used to aid in the diagnosis and risk stratification of such patients [20,21].

\section{Diagnostic Utility of Natriuretic Peptides in Acute Heart Failure}

The utility of BNP in the diagnosis of HF in the emergent setting was revolutionized by Maisel et al. [21]. demonstrating that in 1586 patients presenting to the ED with a chief complaint of dyspnea, a BNP level of $100 \mathrm{pg} / \mathrm{ml}$ carried $90 \%$ sensitivity and $73 \%$ specificity in diagnosing HF. Adding BNP to clinical judgment improved its diagnostic accuracy from 74 to $81 \%$; moreover, BNP was found to be superior to the NHANES and Framingham criteria which are widely used in the diagnosis of HF. Left ventricular systolic dysfunction is often seen on patients with AHF.

The BNP levels have been shown to significantly correlate with systolic dysfunction. In a prospective study comparing neurohormonal markers in assessing left ventricular dysfunction after acute MI, results revealed that BNP had the strongest inverse relation to left ventricular ejection fraction. Patients that developed HF on follow-up (80\%), all had elevated BNP levels, moreover, BNP achieved $84 \%$ sensitivity, $77 \%$ specificity and $95 \%$ negative predictive value in development of left ventricular failure [22].

The multicenter REDHOT trial [23] assessed the perceived severity and clinical decision making among ED physicians with respect to BNP levels obtained at admission. Clinicians often rely on physical examination findings and resolving symptoms to help decide the appropriate time to discharge the patient. The study revealed that there existed a disconnect between perceived severity of illness by ED physicians and severity determined by BNP levels. The study concluded that using BNP levels to aid clinical decision making may prove favorable by limiting length of stay, and improve risk stratification by accurately identifying those at high risk especially if BNP levels were $>200 \mathrm{pg} / \mathrm{ml}$.

Several studies thus far have documented a significant correlation of both BNP and NT-proBNP with left ventricular filling pressures. Iwanaga et al. [24] demonstrated that in 160 patients presenting with HF, BNP levels strongly correlated with left ventricular end-diastolic wall stress $(r=0.887, p<0.001)$, also, the correlation between BNP levels and left ventricular end-diastolic pressure was significant $(r=0.29, p<0.001)$. A substudy of the NP in the community study assessed the prognostic utility of NTproBNP along with echocardiography to predict death and/or re-admissions in an elderly population of 228 participants [25]. Results revealed that patients with high diastolic filling patterns as determined by echocardiography, in addition to elevated NT-proBNP levels, had the worst survival.

Although BNP and NT-proBNP levels are unable to distinguish systolic from diastolic HF, studies have shown that NPs correlate with diastolic wall stress and dysfunction. Tschöpe et al. [26] demonstrated that NT-proBNP levels strongly correlated with isolated diastolic dysfunction and increased severity of diastolic dysfunction $(r=0.67$, $p<0.001)$. The NT-proBNP demonstrated the strongest negative predictive value $(94 \%)$ for diastolic dysfunction.

Prognostic Utility of Natriuretic Peptides in Acute Heart Failure

The prognostic utility of NPs in risk stratification and predicting mortality and morbidity in patients with AHF has been well documented [27, 28]. In an attempt to assess 
if admission BNP levels were predictive of in-hospital mortality in patients with ADHF, BNP levels obtained within $24 \mathrm{~h}$ of presentation in 48,629 patients from the national registry database revealed that elevated BNP levels at admission were significantly associated with in-hospital mortality in patients with ADHF. Those in the highest quartile of BNP levels had the highest in-hospital mortality rate (with $\mathrm{BNP}>1,730 \mathrm{pg} / \mathrm{ml}$ in-hospital mortality was $6 \%$ ) even after adjusting for variables such as age, gender, SBP, BUN, creatinine, sodium, pulse and dyspnea at rest [29]. In a multi-center study of 287 patients presenting to the ED with ADHF receiving AHF treatment, BNP levels measured at admission, $24 \mathrm{~h}$, and at discharge revealed that an absolute BNP reduction of $>46 \%$ at discharge in combination with BNP levels $<300 \mathrm{pg} / \mathrm{ml}$ also at discharge had the most favorable outcomes and least amount of re-hospitalizations and deaths [30•].

The cost-effectiveness of using BNP levels were highlighted in the BASEL study [31] which revealed that testing BNP levels using a rapid bed-side assay in conjunction with physical examination and clinical judgment was able to reduce mean treatment cost and length of stay. Participants were divided into two groups, one which received therapy guided by BNP levels and the control group which received therapy according to existing clinical guidelines. Those in the BNP group also had reduced need for hospitalization and intensive care [31].

Similarly, the utility of NT-proBNP levels in ADHF prognosis achieved substantial results. Bettencourt et al. [32] demonstrated that in 182 patients admitted with ADHF, variations in NT-proBNP levels from admission along with signs of volume overload were independent predictors of adverse outcomes. In order to assess N-terminal proBNP levels in response to treatment, participants were divided into three groups. The groups were those with NT-proBNP levels decreasing with therapy, those with no change in NT-proBNP levels from baseline and those with increasing NT-proBNP levels. The study demonstrated that patients with re-admissions had elevated NT-proBNP levels. The rise in NT-proBNP levels of $>30 \%$ during hospitalization was strongly associated with adverse outcomes.

These studies underline the efficacy of NPs in managing patients with AHF. The NPs response to treatment can be used to titrate therapy in patients with AHF. The NPs provide additional diagnostic and prognostic value and their utility in combination with clinical judgment and physical examination may achieve the most favorable outcomes in treating AHF.

\section{Caveats to Using Natriuretic Peptide}

Interpretation of NP levels in patients with AHF can be sometimes confusing in the presence of other comorbidities and thus other factors affecting NP levels must be considered such as obesity, renal function impairment, presence of atrial fibrillation, and flush pulmonary edema. Moreover, certain NP values lie in the grey-zone requiring a more comprehensive effort in terms of physical examination and clinical testing in order to identify other causes of elevated NP levels as shown in Table 1.

\section{Troponins}

The cardiac troponin complex consists of subunits C, I and $\mathrm{T}$ and are responsible for calcium mediated contraction of cardiac muscle [33]. Cardiac troponin I and $\mathrm{T}$ are specific to cardiac muscle and are released into the bloodstream as a result of myocyte necrosis $[34,35]$. Once in the circulation, cardiac troponins are measurable for 4-10 days before they are cleared by the reticuloendothelial system and renally excreted [36]. Measuring troponin levels in patients with AHF offer information on ongoing myocardial damage, although it must be remembered that multiple other conditions such as local infection, or inflammation, myocyte stretch and other conditions causing cellular death can cause elevated troponin levels [37, 38]. In patients with AHF the mechanism of troponin release may be partly explained by the presence of myocyte stretch due to high filling pressures resulting in troponin leakage and spilling over into the circulation [39]. Several studies have demonstrated the adverse effect of elevated troponin levels in patients hospitalized with AHF. Arenja et al. [40] demonstrated that in 667 patients presenting to the ED with acute dyspnea, diagnostic accuracy of cardiac troponin I (cTnI) in diagnosing AHF achieved an AUC of 0.78 on ROC analysis.

The availability of highly sensitive assays has allowed the detection of even minute levels of troponins which are elevated in the blood after a period of cellular insult [41].

In assessing the prognostic potential of high-sensitivity troponin I in addition to BNP, 144 patients with ADHF had these biomarkers measured at admission, during their inpatient stay and were followed for 90-days to check mortality and HF-related re-admissions [42•]. Results revealed that in hospitalized patients, elevated troponin I along with elevated BNP carried the worst prognosis (HR 15.97, $p=0.007)$ followed by those with low BNP but elevated troponin I (HR 12.94, $p=0.015$ ). Serially measuring highsensitivity troponin levels in in-patients revealed that even the smallest elevation in troponin levels (cut-off $23.35 \mathrm{ng} /$ $\mathrm{ml}$ ) was associated with poor prognosis [42•].

The utility of troponin levels in patients with AHF allows the identification of patients at high risk of mortality due to cardiovascular causes and those at high risk for readmissions. With a large number of patients with ADHF 
Table 1 Factors affecting natriuretic peptide levels in patients with $\mathrm{HF}$ along with "grey-zone" values in diagnosing HF

\begin{tabular}{|c|c|c|}
\hline & BNP & NT-proBNP \\
\hline \multicolumn{3}{|l|}{ Factors affecting NP levels } \\
\hline Pulmonary disease ${ }^{\mathrm{a}}$ & $\uparrow$ & $\uparrow$ \\
\hline Renal disease $^{\mathrm{b}}$ & $\uparrow$ & $\uparrow$ \\
\hline Diastolic dysfunction $^{c}$ & $\uparrow$ & $\uparrow$ \\
\hline Obesity $^{\mathrm{d}}$ & $\downarrow$ & $\downarrow$ \\
\hline Flash pulmonary edema ${ }^{\mathrm{e}}$ & $\downarrow$ & $\downarrow$ \\
\hline Other causes $^{\mathrm{f}}$ & $\downarrow$ & $\downarrow$ \\
\hline \multicolumn{3}{|l|}{ Diagnostic cut-off } \\
\hline HF present $(\mathrm{pg} / \mathrm{ml})$ & $>400$ & $\begin{array}{l}<50 \text { years: }>450 \\
50-75 \text { years: }>900 \\
>75 \text { years: }>1800\end{array}$ \\
\hline HF absent (pg/ml) & $<100$ & $\begin{array}{l}<75 \text { years: } 125 \\
>75 \text { years: } 450\end{array}$ \\
\hline Grey-zone (pg/ml) & $100-400$ & $\begin{array}{l}<50 \text { years: } 300-450 \\
\text { 50-75 years: } 300-900 \\
>75 \text { years: } 300-1800\end{array}$ \\
\hline
\end{tabular}

$B N P$ B-type natriuretic peptide, $N T$-proBNP amino-terminal B-type natriuretic peptide

${ }^{\text {a }}$ In patients with pulmonary disease such as chronic obstructive lung disease or pneumonia, natriuretic peptide levels may be elevated due to right ventricular stress

${ }^{b}$ Patients with worsening renal function often have elevated NP levels due to decreased glomerular filtration of peptides

c Although NPs are unable to distinguish between systolic and diastolic HF, their high negative predictive value make them an effective screening tool in such patients

${ }^{\mathrm{d}}$ In patients with BMI $>35 \mathrm{~kg} / \mathrm{m}^{2}$ low NP levels are possibly due to increased clearance via adipocytes, thus using low cut-off values may improve diagnostic accuracy in such patients

e In the acute setting, NP levels may be deceivingly low, thus care should be taken to serially monitor NP levels in these patients

f Patients with HF due to valvular disorders, pericardial tamponade/ constriction, which are upstream from the left ventricle, may also exhibit low NP levels. When suspecting HF, BNP $<100 \mathrm{pg} / \mathrm{ml}$ "rules out" HF whereas BNP $>100 \mathrm{pg} / \mathrm{ml}$ "rules in" HF. Similarly for NTproBNP levels, age-stratified diagnostic levels can be used to "rule in" or "rule out" HF

seeking re-admissions, monitoring troponin levels may provide a way to gauge treatment efficacy and allow titration of HF therapy.

\section{Markers of Comorbidities Seen in Acute Heart Failure Patients}

\section{Procalcitonin}

In humans procalcitonin release is induced by the bacterial endotoxin making procalcitonin an effective marker for ongoing bacterial infection [43-45]. Encoded by the CALC
1 gene, procalcitonin is a 116 amino acid containing peptide released from parenchymal tissue of liver, lungs, brain, adipose and formed by the cleavage of its precursor protein [44-47]. In healthy individuals procalcitonin is produced primarily in thyroidal c-cells where it undergoes further processing to yield mature biologically active calcitonin $[45,46]$. The AHF patients often present with pulmonary bacterial infections such as pneumonia, which is difficult to detect with conventional tests such as chest $\mathrm{x}$-ray and blood testing. This leads to misdiagnosis of underlying pneumonia since the majority of these patients with AHF present in the ED with a chief complaint of dyspnea, which is difficult to distinguish using conventional blood tests and a chest x-ray $[48 \cdot \bullet]$.

Maisel et al. [48••] demonstrated that procalcitonin was useful in identifying patients with AHF and underlying pneumonia. The study evaluated patients with AHF who presented to the ED and identified those with pneumonia and those receiving antibiotic use, following which procalcitonin levels were measured in these individuals using sensitive assays. Median Procalcitonin concentrations in patients with pneumonia were $0.18 \mathrm{ng} / \mathrm{ml}$ (IQR 0.07-0.58) and $0.07 \mathrm{pg} / \mathrm{ml}$ (IQR 0.05-0.12) for those without pneumonia. Using procalcitonin levels improved diagnostic accuracy and physical estimates of diagnosing pneumonia in patients with AHF. The study also suggested that, if procalcitonin levels were $>0.21 \mathrm{ng} / \mathrm{ml}$, antibiotic use improved survival in these patients suggesting the possibility of procalcitonin guided antibiotic use in AHF patients.

\section{Neutrophil Gelatinase Associated Lipocalin}

Neutrophil gelatinase associated lipocalin (NGAL) contains a 178 amino acid structure and is secreted from the neutrophils and epithelial proximal tubules in response to ischemia [49]. Studies have shown that NGAL levels are superior to creatinine in predicting early renal ischemia [49-51]. Physiologically, once released, NGAL performs multiple functions. The NGAL functions as an iron-binding siderophore acting as an iron-transport protein to prevent bacteria from utilizing iron stores for cellular growth. The NGAL also triggers nephrogenesis by stimulating conversion of mesenchymal cells into kidney epithelia thus enhancing epithelial phenotype. Finally, NGAL facilitates recycling of iron by its ability to be endocytosed by the proximal tubule thus limiting iron-mediated toxicity [49-51].

In patients with AHF, worsening renal function can be a result of multiple factors such as administration of nephrotoxic agents like intravenous contrast, or over diuresing leading to acute kidney injury [52, 53]. The NGAL is released into the circulation within $2 \mathrm{~h}$ of acute kidney 
injury and serves as an excellent marker for worsening renal function. In a single center prospective study, NGAL levels were obtained at admission, and at 48 and $72 \mathrm{~h}$ in patients diagnosed with ADHF. Results revealed that patients with worsening renal function (defined as serum creatinine $>0.3 \mathrm{mg} / \mathrm{dl}$ from admission) had higher admission NGAL levels (194 vs. $128 \mathrm{ng} / \mathrm{ml}, p=0.01$ ) whereas serum creatinine levels were in the normal range. The NGAL level $>140 \mathrm{ng} / \mathrm{ml}$ yielded $86 \%$ sensitivity, $54 \%$ specificity and $86 \%$ negative predictive value in detecting worsening renal function [53].

The GALLANT trial [54] demonstrated that NGAL levels along with BNP served as an excellent indicator of adverse outcomes in 188 patients presenting to the emergency department with ADHF. On Cox regression analysis in assessing re-admission and all-cause mortality at 30-days, log NGAL levels were significant predictors of adverse events along with BNP where as neither serum creatinine nor estimated glomerular filtration rate achieved significance. Further analysis revealed that those with elevated NGAL levels ( $>100 \mathrm{ng} / \mathrm{ml})$ but lower BNP levels $(<330 \mathrm{pg} / \mathrm{ml})$ had HR of $9.95(p=0.036)$ and HR of 16.85 $(p=0.006)$ if both markers were elevated.

Creatinine is a poor indicator of early developing renal injury and, in patients with AHF presenting to the ED, measuring NGAL levels may help identify those patients at risk of worsening of renal function and, thus, may prevent overuse of pharmacological/nephrotoxic agents in these patients. Furthermore, patients with elevated BNP and NGAL levels at discharge must be identified in order to effectively tailor treatment in these patients and prevent progression of $\mathrm{HF}$ and protect renal function.

\section{Emerging Biomarkers in Acute Heart Failure}

\section{Copeptin}

Copeptin is a glycosylated peptide with a 39 amino acid structure secreted in equimolar concentrations with arginine vasopressin (AVP) from the hypothalamus [55]. Studies have shown that due to limited availability, and rapid clearance from the circulation, measuring AVP in the plasma is difficult $[56,57]$. Copeptin is secreted from the AVP precursor protein along with AVP and neurophysin II, and due to its stability in the plasma, measuring copeptin levels are beneficial. The main function of AVP is in maintaining hemodynamic stability by reabsorbing water at the renal tubules in direct response to changing plasma volume and osmolality [57-59].

The large-scale study to date evaluating prognostic utility of copeptin in AHF patients was conducted by Maisel et al. [60] demonstrating an increased risk of 90-day mortality, high re-admission rate and $\mathrm{ED}$ visits related to $\mathrm{HF}$ in patients with elevated copeptin levels. Copeptin's effects are mediated via VI and V2 receptors leading to progression of left ventricular hypertrophy and myocardial remodeling [5759]. The study also demonstrated a lack of correlation between sodium and copeptin in this study, possibly explained by the complex underlying physiology of water and sodium regulation. On univariate analysis, copeptin along with NT-proBNP were significant predictors of mortality with HR 2.87 for copeptin and HR 3.46 for NT-proBNP ( $p<0.001$ for both). On multivariate analysis, sodium along with copeptin and NT-proBNP were significant predictors of mortality (copeptin: $p=0.007$, HR 2.115; sodium: $p<0.001$, HR 2.739; NT-proBNP: $p<0.003$, HR, 2.383) Thus, elevated copeptin levels in patients with AHF is a definite sign of poor outcome, especially in the setting of hyponatremia, and adequate measures must be taken to prevent progression of HF in these patients [60].

\section{Galectin-3}

Galectin-3 belongs to the family of $\beta$-galactosides and in patients with $\mathrm{HF}$ is responsible for mediating cardiac fibrosis and ventricular dysfunction by activating fibroblast production [61, 62]. In an effort to demonstrate the diagnostic and prognostic utility of Galectin-3 along with NPs, Kimmenade et al. [63] showed that in 599 patients with acute dyspnea presenting to the ED, Galectin-3 levels independently predicted 60-day mortality (OR 10.3, $p<0.01$ ) yielding an AUC of 0.74 superior to NT-proBNP and apelin on ROC analysis. In diagnosing AHF in dyspneic patients, galectin-3 performed second best to NT-proBNP on ROC analysis producing an AUC of 0.72 vs. 0.94 for NT-proBNP and 0.52 for apelin, an endogenous inotropic peptide. Although studies exploring the utility of Galectin-3 in patients with AHF are upcoming, its role in progression of HF remains intriguing and can be seen as a potential for targeting treatment in order to halt the progression of HF.

\section{Conclusion}

Biomarkers in AHF reflect distinct pathophysiological events and play a very specific role in the diagnosis and risk stratification of these patients. They are an essential tool, and when used as an adjunct to ancillary testing, provide the best outcomes for the patients. The NP continue to be extremely useful in identifying volume overload and when used in conjunction with procalcitonin can be useful in identifying patients with superimposed pneumonia. Interpreting NP levels must be undertaken with care especially in patients with underlying pulmonary/renal disease. Moreover, in patients with "grey-zone" values, 
care must be taken in further investigation as to the cause of elevated biomarker levels. The utility of NGAL seems to be in identifying sub-clinical ischemia especially in the acute setting, wherein creatinine seems to be a poor marker of worsening renal function. Furthermore, it can be used together with BNP can help identify patients with volume overload in need of diuretic treatment so as to prevent overdiuresis and avoid further worsening of renal function. Finally the new and upcoming biomarkers provide more information on cardiac remodeling and are indicative of AHF progression, monitoring these biomarkers in hospitalized patients with AHF may help identify those at high risk of worsening HF. Biomarkers allow monitoring response to treatment. Moreover, using multiple biomarkers in bio-monitoring and guiding treatment may prove beneficial in improving mortality associated with AHF.

Disclosure No potential conflicts of interest relevant to this article were reported.

\section{References}

Papers of particular interest, published recently, have been highlighted as:

- Of importance

•• Of major importance

1. O'Connor CM, Abraham WT, Albert NM, et al. Predictors of mortality after discharge in patients hospitalized with heart failure: an analysis fro the organized program to initiate lifesaving treatment in hospitalized patients with heart failure (OPTIMIZEHF). Am Heart J. 2008;156:662-73.

2. Weintraub NL, Collins SP, Pang P, et al. Acute heart failure syndromes: emergency department presentation, Treatment, and disposition: current approaches and future aims. Circulation. 2010;122:1975-96.

3. McMurray JJV, Adamopoulos S, Anker SD, et al. ESC Guidelines for the diagnosis and treatment of acute and chronic heart failure 2012. Eur Heart J. 2012;33:1787-847.

4. Fonarow GC, Heywood JT, Heidenreich PA, et al. Temporal trends in clinical characteristics, treatments and outcomes for heart failure hospitalizations, 2002 to 2004: findings from acute decompensated national registry (ADHERE). Am Heart J. 2007; 153:1021-8.

5. Follath F, Yilmaz MB, Delgado JF, et al. Clinical presentation, management and outcomes in the acute heart failure global survey of standard treatment (ALARM-HF). Intensive Care Med. 2011;37:619-26.

6. Gheorghiade M, Zannad F, Sopko G, et al. Acute heart failure syndromes current state and framework for future research. Circulation. 2005;112:3958-68.

7. De Luca L, Fonarow GC, Adams KF Jr, Mebazaa A, et al. Acute heart failure syndromes: clinical scenarios and pathophysiologic targets for therapy. Heart Fail Rev. 2007;12:97-104.

8. Metra M, Felker GM, Zaca V, et al. Acute heart failure: multiple clinical profiles and mechanisms require tailored therapy. Int $\mathbf{J}$ Cardiol. 2010;144:175-9.
9. Spinar J, Parenica J, Vitovec J, et al. Baseline characteristics and hospital mortality in the acute heart failure database (AHEAD) main registry. Crit Care. 2011;15:R291.

10. Cotter G, Felker M, Adams KF, et al. The pathophysiology of acute heart failure-is it all about fluid accumulation? Am Heart J. 2008;155:9-18.

11. Smith GL, Lichtman JH, Bracken MB, et al. Renal impairment and outcomes in heart failure: systematic review and meta-analysis. J Am Coll Cardiol. 2006;16:1987-96.

12. Rudiger A, Harjola VP, Muller A, et al. Acute heart failure: clinical presentation, one-year mortality and prognostic factors. Eur J Heart Fail. 2005;7:662-70.

13. Morrow DA, De Lemos JA. Benchmarks for the assessment of novel cardiovascular biomarkers. Circulation. 2007;115:949-52.

14. Hlatky MA, Greenland P, Arnett DK, et al. Criteria for evaluation of novel markers of cardiovascular risk. Circulation. 2009;119: 2408-16.

15. Levin ER, Gardner DG, Samson WK. Natriuretic peptides. N Engl J Med. 1998;339:321-8.

16. Mueller T, Gegenhuber A, Poelz W, Haltmayer M. Diagnostic accuracy of $\mathrm{B}$ type natriuretic peptide and amino terminal proBNP in the emergency diagnosis of heart failure. Heart. 2005;91:606-12.

17. Clerico A, Recchia FA, Passino C, Emdin M. Cardiac endocrine function is an essential component of the homeostatic regulation network: physiological and clinical implications. Am J Physiol Heart Circ Physiol. 2006;290:H17-29.

18. McCullough PA, Omland T, Maisel AS. B-type natriuretic peptides: a diagnostic breakthrough for clinicians. Rev Cardiovasc Med. 2003;4:72-80.

19. De Bold AJ, Borenstein HB, Veress AT, Sonnenberg H. A rapid and potent natriuretic response to intravenous injection of atrial myocardial extract in rats. Life Sci. 1981;28:89-94.

20. Misono KS, Philo JS, Arakawa T, et al. Structure, signaling mechanism and regulation of the natriuretic peptide receptor guanylate cyclase. FEBS J. 2011;278:1818-29.

21. Maisel AS, Krishnaswamy P, Nowak R, et al. Rapid measurement of B-type natriuretic peptide in the emergency diagnosis of heart failure. N Engl J Med. 2002;347:161-7.

22. Richards AM, Nicholls MG, Yandle TG, et al. Neuroendocrine prediction of left ventricular function and heart failure after acute myocardial infarction. Heart. 1999;81:114-20.

23. Maisel A, Hollander JE, Guss D, et al. Primary results of the rapid emergency department heart failure outpatient trial (REDHOT). J Am Coll Cardiol. 2004;44:1328-33.

24. Iwanaga Y, Nishi I, Furuichi S, et al. B-type natriuretic strongly reflects diastolic wall stress in patients with chronic heart failure: comparison between systolic and diastolic heart failure. J Am Coll Cardiol. 2006;47:742-8.

25. Whalley GA, Wright SP, Pearl A, et al. Prognostic role of echocardiography and brain natriuretic peptide in symptomatic breathless patients in the community. Eur Heart J. 2008;29:509516.

26. Tschöpe C, Kašner M, westerman D, et al. The role of NTproBNP in the diagnostics of isolated diastolic dysfunction: correlation with echocardiographic and invasive measurements. Eur Heart J. 2005;26:2277-84.

27. Gegenhuber A, Mueller T, Dieplinger B, Poelz W, et al. B-type natriuretic peptide and amino terminal proBNP predict one-year mortality in short of breath patients independently of the baseline diagnosis of acute destabilized heart failure. Clin Chim Acta. 2006;370:174-9.

28. Wood MJ, Krauser DG, Chen AA, et al. NT-proBNP levels, echocardiographic findings, and outcomes in breathless patients: results from the ProBNP Investigation of Dyspnoea in the Emergency Department (PRIDE) echocardiographic substudy. Eur Heart J. 2006;27:839-45. 
29. Fonarow GC, Peacock WF, Phillips CO, et al. Admission B-type natriuretic peptide levels and in-hospital mortality in Acute decompensated heart failure. J Am Coll Cardiol. 2007;49:1943-50.

30. - Di Somma S, Magrini L, Pittoni V, et al. In-hospital percentage BNP reduction is highly predictive for adverse events in patients admitted for acute heart failure: the Italian RED study. Crit Care. 2010;14:R116. This is a paper that demonstrated the utility of $B N P$ assessment at hospital admission and discharge in order to verify the percent change and the possibility of hospital readmission in patients with acute heart failure.

31. Mueller C, Scholer A, Laule-Killian K, et al. Use of B-type natriuretic peptide in the evaluation and management of acute dyspnea. N Engl J Med. 2004;350:647-54.

32. Bettencourt P, Azevedo A, Pimenta J, et al. N-terminal-pro-brain natriuretic peptide predicts outcome after hospital discharge in heart failure patients. Circulation. 2004;110:2168-74.

33. Morrow DA, Canon CP, Jesse RL, et al. National Academy of Clinical Biochemistry Laboratory Medicine Practice Guidelines: clinical characteristics and utilization of biochemical markers in acute coronary syndromes. Clin Chem. 2007;53:552-74.

34. Daubert MA, Jeremias A. The utility of troponin measurement to detect myocardial infarction: review of the current findings. Vasc Health Risk Manag. 2010;6:691-9.

35. Gerhardt W, Nordin G, Ljungdahl L. Can troponin T replace CK MBmass as "gold standard" for acute myocardial infarction ("AMI")? Scand J Clin Lab Invest Suppl. 1999;230:83-9.

36. Diris JH, Hackeng CM, Kooman JP, Pinto YM, Hermens WT, van Dieijen-Visser MP. Impaired renal clearance explains elevated troponin $\mathrm{T}$ fragments in hemodialysis patients. Circulation. 2004;109(1):23-5.

37. McDonough JL, Arrell DK, Van Eyk JE. Troponin I degradation and covalent complex formation accompanies myocardial ischemia/reperfusion injury. Circ Res. 1999;84(1):9-20.

38. Hessel MH, Atsma DE, van der Valk EJ, Bax WH, Schalij MJ, van der Laarse A. Release of cardiac troponin I from viable cardiomyocytes is mediated by integrin stimulation. Pflugers Arch. 2008;455(6):979-86.

39. Thygesen K, Mair J, Katus H, Plebani M, Venge P, Collinson P, et al. Recommendations for the use of cardiac troponin measurement in acute cardiac care. Eur Heart J. 2010;31(18):2197-204.

40. Arenja N, Reichlin T, Drexler B, et al. Sensitive cardiac troponin in the diagnosis and risk stratification of acute heart failure. J Intern Med. 2012;271:598-607.

41. Weber M, Bazzino O. Navarro Estrada JL, de Miguel R, Salzberg $\mathrm{S}$, Fuselli JJ, et al. Improved diagnostic and prognostic performance of a new high-sensitive troponin $\mathrm{T}$ assay in patients with acute coronary syndrome. Am Heart J. 2011;162(1):81-8.

42. - Xue Y, Clopton P, Peacock WF, Maisel AS. Serial changes in high-sensitive troponin I predict outcome in patients with decompensated heart failure. Eur J Heart Fail. 2011;13:37-42. This is an important paper outlining the importance of serial troponin assessments in patients with Acute Heart Failure in order to stratify the risk of events.

43. Russworm S, Wiederhold M, Oberhoffer M, et al. Molecular Aspects and Natural Source of Procalcitonin. Clin Chem Lab Med. 1999;37:789-97.

44. Ittner L, Born W, Rau B, et al. Circulating Procalcitonin and cleavage products in septicemia compared with medullary thyroid carcinoma. Eur J Endocrinol. 2002;147:727-31.

45. Meisner M. Pathobiochemsitry and clinical use of procalcitonin. Clin Chim Acta. 2007;323:17-29.

46. Carrol ED, Thomson APJ, Hart CA. Procalcitonin as a marker of sepsis. Int J Antimicrob Agents. 2002;20:1-9.

47. Linscheid P, Seboek D, Schaer DJ, Zulewski H, et al. Expression and secretion of Procalcitonin and calcitonin gene-related peptide by adherent monocytes and by nacrophage-activated adipocytes. Crit Care Med. 2004;32:1715-21.

48. •• Maisel A, Neath SX, Landsberg J, Mueller C, et al. Use of procalcitonin for the diagnosis of pneumonia in patients presenting with a chief complaint of dyspnea: results from the BACH (biomarkers in acute heart failure) trial. Eur J Heart Fail. 2012;14:278-86. This is a very important paper from a subanalysis of the BACH trial suggesting the importance of the multimarkers approach (procalcitonin + Natriuretic peptides) in patients with acute shortness of breath in order to distinguishing underlying causes: acute heart failure, pneumonia or both).

49. Mishra J, Ma Q, Kell C, et al. Kidney NGAL is a novel early marker of acute injury following transplantation. Pediatr Nephrol. 2006;21:856-63.

50. Mishra J, Dent C, Tarabishi R, et al. Neutrophil gelatinase associated lipocalin (NGAL) as a biomarker for acute renal injury after cardiac surery. Anesthesiology. 2006;105:485-91.

51. Mori K, Lee HT, Rapoport D, et al. Endocytic delivery of lipocalin-siderophore-iron complex rescues the kidney from ischemia-reperfusion injury. J Clin Invest. 2005;115:610-21.

52. Damman K, Navis G, Voors AA, et al. Worsening renal function and prognosis in heart failure: systematic review and metaanalysis. J Card Fail. 2007;13:599-608.

53. Aghel A, Shreshtha K, Mullens W, et al. Serum neutrophil gelatinase-associated lipocalin (NGAL) in predicting worsening renal function in acute decompensated heart failure. J Card Fail. 2010;16:49-54.

54. Maisel AS, Mueller C, Fitzgerald R, et al. Prognostic utility of plasma neutrophil gelatinase associated lipocalin in patients with acute heart failure: the NGAL evaluation along with B-type natriuretic peptide in acutely decompensated heart failure (GALLANT) trial. Eur J Heart Fail. 2011;13:846-51.

55. Stoiser B, Mortl D, Hulsmann M, et al. Copeptin, a fragment of the vasopressin precursor, as a novel predictor of outcome in heart failure. Eur J Clin Invest. 2006;36:771-8.

56. Morgenthaler NG, Struck J, Alonso C, Bergmann A. Assay for the measurement of copeptin, a stable peptide derived from the precursor of vasopressin. Clin Chem. 2006;52:112-9.

57. Neuhold S, Huelsmann M, Strunk G, et al. Comparison of copeptin, B-type natriuretic peptide, and amino-terminal pro-B-type natriuretic peptide in patients with chronic heart failure: prediction of death at different stages of the disease. J Am Coll Cardiol. 2008;52:266-72.

58. Von Haehling S, Anker SD, Voors amino acid, et al. C-terminal provasopressin (copeptin) is a strong prognostic marker in a patient with heart failure after an acute myocardial infarction: results from the OPTIMamino acidL study. Eur Heart J. 2009;30:1187-94.

59. Kelly D, Squire IB, Khan SQ, et al. C-terminal provasopressin (copeptin) is associated with left ventricular dysfunction and clinical heart failure in survivors of myocardial infarction. J Card Fail. 2008;14:739-45.

60. Maisel A, Xue Y, Shah K, et al. Increased 90-day mortality in patients with acute heart failure with elevated copeptin. Circ Heart Fail. 2011;4:613-20.

61. Sharma UC, Pokharel S, Van Brakel TJ, et al. Galectin-3 marks activated macrophages in failure-prone hypertrophied hearts and contributes to cardiac dysfunction. Circulation. 2004;110:3121-8.

62. De Boer RA, Voors AA, Muntendam P, et al. Galectin-3: a novel mediator of heart failure development and progression. Eur $\mathrm{J}$ Heart Fail. 2009;11:811-7.

63. Van Kimmenade RR, Januzzi JL, Ellinor PT, et al. Utility of amino-terminal pro-brain natriuretic peptide, galectin-3, and apelin for the evaluation of patients with acute heart failure. J Am Coll Cardiol. 2006;48:1217-24. 